\title{
PREVALENCIA DE FACTORES DE RIESGO CARDIOMETABÓLICOS EN DOCENTES Y ESTUDIANTES DE POSGRADO DE LA FUCS
}

\section{SEGUNDO SEMESTRE 2010}

José Fernando Orduz Sánchez MD*, Narda Piedad Chacón Peña MD**, Gilma Eleonora Rozo Acuña MD**, Dairo Fernando Beltrán Talero MD**, Juan Carlos Ospina Martínez MD***

\section{Resumen}

Las enfermedades cardiovasculares son la principal causa de muerte en todo el mundo y de ellas la hipertensión, la hipercolesterolemia y el tabaquismo son las tres cuartas partes de los casos. Ante su alta prevalencia y carga de morbilidad, surgió la inquietud de determinar si en la población médica se mantienen estos mismos niveles, al ser el pilar de la atención primaria. Objetivo: describir la prevalencia de los factores de riesgo cardiometabólico en estudiantes de posgrado y docentes de la facultad de medicina de la FUCS. Metodología: estudio de corte transversal. En el segundo semestre 2010 se realizó valoración médica mediante autodiligenciamiento de un cuestionario y la evaluación antropométrica. Se tomaron muestras para glicemia y perfil lipídico basal para calcular el riesgo cardiovascular por Framingham. Resultados: tasa de respuesta $61.2 \%(345 / 564)$, género masculino $61.7 \%$, docentes $32.7 \%$, estudiantes $67.3 \%$, edad promedio (años) 34.3 (DE:9.1), prevalencia de obesidad abdominal 33.6\%, sobrepeso 35.9, obesidad (IMC) 7.2\%, tabaquismo 16.2\%, síndrome metabólico 17.4\%, hipertrigliceridemia 42.\%, HDL bajo 41.5\%, sedentarismo $80.2 \%$ y nivel de riesgo cardiovascular latente $79 \%$, intermedio $18.9 \%$ y alto $2 \%$.

Palabras clave: riesgo cardiometabólico, riesgo cardiovascular, docentes, estudiantes de posgrado, facultad de medicina.

Abreviaturas: ECV, enfermedades cardiovasculares.

\section{PREVALENCE OF CARDIOMETABOLIC RISK WITHIN FUCS GRADUATE PROGRAM FACULTY MEMBERS AND STUDENTS SECOND SEMESTER 2010}

\begin{abstract}
Cardiovascular diseases are the leading cause of deaths worldwide and three-quarters of all cases are attributable to hypertension, hypercholesterolemia and smoking. The question on determining if similar levels of cardiovascular diseases are maintained within the medical population as the pillar of primary care was brought up due to their high prevalence and morbidity burden. Objective: to describe the prevalence of cardiometabolic risk factors within graduate program faculty members and students at the FUCS School of Medicine. Methodology: a cross-sectional study. A medical evaluation was performed by filling a questionnaire and conducting an anthropometric evaluation
\end{abstract}

Fecha recibido: enero 16 de 2012 - Fecha aceptado: marzo 23 de 2012

* Magister en Salud Pública. Magister en Medicinas Alternativas, Instructor Asociado, Fundación Universitaria de Ciencias de la Salud, Bogotá DC. Colombia.
* Especialista en Medicina Familiar, Instructor Asistente, Fundación Universitaria de Ciencias de la Salud, Bogotá DC. Colombia.

*** Residente III de Medicina Familiar, Fundación Universitaria de Ciencias de la Salud, Bogotá DC. Colombia. . 
during the second semester of 2010. Blood samples for fasting glucose and baseline lipid profile were drawn to estimate the cardiovascular risk using the Framingham scale. Results: response index: 61.2\% (345/564), males: 61.7\%, faculty members: $32.7 \%$, students: $67.3 \%$, mean age (years): 34.3 (SD: 9.1), prevalence of abdominal obesity: $33.6 \%$, overweight: 35.9, obesity (BMI): 7.2\%, smoking: 16.2\%, metabolic syndrome: 17.4\%, hypertriglyceridemia 42. \%, low

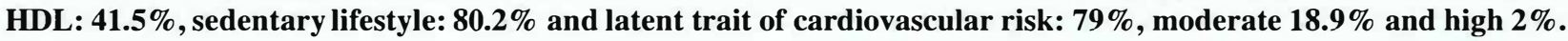

Key words: cardiometabolic risk, cardiovascular risk, faculty, graduate program students, school of medicine.

\section{Introducción}

Las ECV representan la principal causa de morbimortalidad en el mundo, en especial en países en vía de desarrollo. Se calcula que para el 2015 serán responsables de la muerte de más de 20 millones de personas. ${ }^{1}$ Según el informe de estadísticas de salud de las Américas de 2006, la cardiopatía isquémica y las enfermedades cerebrovasculares encabezan las causas de muerte. ${ }^{2}$ Para el 2005 en Colombia la tasa de mortalidad por enfermedades cardiocerebrovasculares fue 130.2 por 100.000 habitantes; en mayores de 45 años la muerte por causa coronaria 265.9, por afecciones cerebrovasculares 136.4 y en diabetes mellitus 73 , todas las cifras por 100.000 habitantes. $^{3}$

Estas enfermedades tienen relación directa con múltiples factores de riesgo, algunos modificables (hipertensión arterial, obesidad, tabaquismo, dislipidemia, diabetes, inactividad física y síndrome metabólico) y otros no (edad, género y genética). De acuerdo al estudio CARMELA la prevalencia de los factores de riesgo cardiometabólico en la ciudad de Bogotá es: diabetes mellitus tipo $28.1 \%$, hipercolesterolemia $12 \%$, hipertensión arterial $13.4 \%$, obesidad $18.0 \%$, síndrome metabólico $20.4 \%$ y tabaquismo $22.2 \% .^{4}$

Muchos estudios analizan la problemática de la ECV a nivel mundial y nacional para la salud pública, pero no se evidencian estudios profundos que aporten prevalencia de factores de riesgo cardiometabólico en la población de estudiantes de posgrado y docentes de facultades de medicina, quienes son el pilar de actividades de promoción de salud y prevención de la enfermedad, en el ámbito de la atención primaria en salud.

Se conoce que la prevalencia de factores de riesgo cardiovascular en profesionales de la salud, según el estudio realizado en el Hospital Ramón González Valencia en Bucaramanga, Colombia en 2004, es: sedentarismo (82.4\%), seguido por hipertensión arterial (54\%), sobrepeso (46.4\%), obesidad central (40.3\%), dislipidemia (24\%) y tabaquismo (10\%). ${ }^{5}$ Se ve cómo esta población cuenta en su mayoría con factores de riesgo cardiovascular modificables, que al ser intervenidos pueden impactar en forma positiva los perfiles de morbimortalidad, mejorando por ende su calidad de vida y el manejo de las actividades de mantenimiento de la salud de sus pacientes. Algunos estudios han mostrado cómo los hábitos de los profesionales de la salud influyen de manera positiva o negativa en la percepción y modificación de dichos hábitos por parte de sus pacientes. ${ }^{6,7}$

En la Fundación Universitaria de Ciencias de la Salud (FUCS) por medio de su facultad de medicina, el programa de posgrado de medicina familiar y el departamento de salud pública se diseñó el presente estudio para determinar el riesgo cardiometabólico en la población docente y en los estudiantes de posgrado. Esto permitirá construir una línea de base que genere intervenciones individuales y colectivas en el marco de un proyecto de universidad saludable con enfoque en medicina familiar y así lograr pautas positivas y modificaciones en los estilos de vida, encaminados a mejorar tanto la calidad de vida de docentes y estudiantes de posgrado de la FUCS, como las consejerías y pautas de educación en factores de riesgo para los propios pacientes.

\section{Métodos}

Diseño de la Investigación: estudio observacional descriptivo de corte transversal que se desarrolló en el segundo semestre del 2010, en el que se invitó a participar a toda la población de docentes y estudiantes de 
posgrado de la facultad de medicina de la FUCS través de diferentes medios de comunicación internos de la universidad. Los criterios de inclusión fueron: para los docentes tener un contrato laboral con la FUCS o las sociedades con actividad académica de la institución, para los estudiantes que cursaran posgrado de la facultad de medicina, especialidades o subespecialidades y estar matriculados en el segundo período académico de 2010. Se consideró como criterio de exclusión ser estudiante de posgrado perteneciente a especialidades administrativas y/o diplomados, teniendo en cuenta que se trata de una población flotante en la institución con tiempo limitado de estancia.

El instrumento utilizado para la recolección de datos y el formato del consentimiento informado fueron revisados y aprobados por el Comité de Investigaciones y Ética de la facultad de Medicina de la FUCS y del Hospital de San José. Previo diligenciamiento del consentimiento informado se realizó la consulta médica, donde se registraron variables como edad, género, especialidad, antecedentes personales y familiares de ECV, nivel de actividad física y tabaquismo a través de un cuestionario autodiligenciado, seguido de un examen físico donde se tomaron medidas antropométricas (tensión arterial, talla, peso, perímetro abdominal e índice de masa corporal), que fueron realizadas por personal médico y de enfermería entrenados, con la correspondiente estandarización de técnicas y calibración de los equipos utilizados.

Después se obtuvieron muestras de sangre en ayunas con el fin de determinar niveles de glicemia, colesterol total, HDL y LDL y triglicéridos, las cuales fueron procesadas en el laboratorio clínico del Hospital de San José. Basados en los datos obtenidos se categorizó el nivel de riesgo cardiovascular utilizando los valores de Framingham. Los resultados se dieron a conocer a cada uno de los participantes de manera individual y confidencial, entregando en medio físico la transcripción dada por el laboratorio. De igual manera se entregó en sobre personalizado las recomendaciones específicas correspondientes al nivel de riesgo cardiovascular detectado. Para el propósito de esta investigación no fueron objeto de análisis las recomendaciones o intervenciones generadas.
Los datos obtenidos se registraron en EXCEL 2007. Para el análisis descriptivo se utilizó el programa STATA 10 y para el análisis multivariado SPAD 7. Las variables cualitativas se presentaron en frecuencias absolutas y porcentajes. Las cuantitativas se informaron como promedio, desviación estándar y valor mínimo y máximo. Cuando la naturaleza de los datos lo requirieron, se reportaron medianas con el rango intercuartílico.

\section{Resultados}

Participaron 345 personas que corresponde al $61.2 \%$ de la población, de los cuales $61.7 \%(\mathrm{n}=213)$ fueron de género masculino, $32.7 \%(\mathrm{n}=113)$ docentes y $67.3 \%$ $(\mathrm{n}=232)$ estudiantes de posgrado. La edad promedio fue 34 años (24-76) (Tabla 1).

\section{Tabla I. Características de la población}

\begin{tabular}{|c|c|c|}
\hline Población & \multicolumn{2}{|c|}{$\mathrm{n}=345$} \\
\hline Edad, años promedio (DE) & 34.3 & $(9.1)$ \\
\hline mínimo - máximo & 24 & 76 \\
\hline \multicolumn{3}{|l|}{ Género } \\
\hline masculino, n (\%) & 213 & $(61.7)$ \\
\hline \multicolumn{3}{|l|}{ Vinculación } \\
\hline docente, n (\%) & 113 & (32.7) \\
\hline estudiante posgrado, n (\%) & 232 & (67.3) \\
\hline \multicolumn{3}{|l|}{ Estado civil } \\
\hline soltero, n (\%) & 187 & $(54.2)$ \\
\hline casado, n (\%) & 128 & (37.0) \\
\hline unión libre, n (\%) & 14 & $(4.1)$ \\
\hline separado, n (\%) & 14 & $(4.1)$ \\
\hline viudo, $n(\%)$ & 2 & $(0.6)$ \\
\hline \multicolumn{3}{|l|}{ Estrato socioeconómico (\%) } \\
\hline 1 & & \\
\hline 2 & I & $(0.3)$ \\
\hline 3 & 67 & $(19.4)$ \\
\hline 4 & 140 & $(40.6)$ \\
\hline 5 & 71 & (20.6) \\
\hline 6 & 66 & $(19.1)$ \\
\hline
\end{tabular}


Según registro de la decanatura la población de estudiantes de posgrado para el tiempo objeto de estudio era de 349 residentes y de acuerdo con recursos humanos de la FUCS la población de docentes de 215 , para un total de 564 personas potenciales, obteniendo una participación de 345 sujetos.

$\mathrm{Al}$ analizar la prevalencia de los factores de riesgo cardiometabólicos distribuidos por género se encuentra que la obesidad abdominal definida por criterios IDF (International Diabetes Federation-perímetro abdominal mayor $\geq 90 \mathrm{~cm}$ en hombres y $\geq 80 \mathrm{~cm}$ en mujeres), predominó en hombres $(45.5 \%, \mathrm{n}=97)$ más que en mujeres $(14.4 \%, \mathrm{n}=19)$. De igual manera el porcentaje de sobrepeso y obesidad por IMC fue de $59.2 \%(n=126)$ en los primeros y de $17.4 \%(n=23)$ en las segundas (Tabla 2).

La mediana del colesterol sérico se ubicó en $201 \mathrm{mg} /$ $\mathrm{dl}$ en hombres (RIQ 183-122) frente a $190 \mathrm{mg} / \mathrm{dl}$ en mujeres (RIQ 167-210). La hipertrigliceridemia se encontró en los primeros $55.8 \%(n=119)$ contra $20.6 \%$ $(\mathrm{n}=27)$. El HDL mostró una mediana de $40 \mathrm{mg} / \mathrm{dl}$ en ellos (RIQ 36-49) y de 57 mg/dl en ellas (RIQ 47-65) evidenciándose que el $41.4 \%$ de la población cursa con niveles de HDL bajos. La mediana para LDL fue de $120 \mathrm{mg} / \mathrm{dl}$ en hombres (RIQ 100-150) contra 110 mg/dl (RIQ 90-130) (Tabla 2).

En relación con la glicemia alterada en ayunas encontramos una prevalencia del $2 \%$ en la población, siendo esta mayor en hombres $(2.8 \%, \mathrm{n}=5)$ que en mujeres $(1.5 \%, n=2)$. Es de anotar que uno de los participantes del estudio presentaba diagnóstico de diabetes mellitus tipo 2 previo al ingreso (Tabla 2). En cuanto al tabaquismo la prevalencia encontrada fue de $18.3 \%$ en hombres $(n=39)$ y del $12.9 \%$ en mujeres $(n=17)$. El $80.2 \%$ de la población se identificó como sedentaria $(n=277)$, siendo esta condición mas prevalente en mujeres $(87.1 \%, \mathrm{n}=115)$ y estudiantes de postgrado (84.5\%, n=196) (Tabla 2).

El síndrome metabólico según criterios últimos IDF ( tres de las cinco siguientes condiciones: perímetro abdominal $\geq 90 \mathrm{~cm}$ en hombres $\mathrm{y} \geq 80 \mathrm{~cm}$ en mujeres, glicemia en ayunas $\geq 100 \mathrm{mg} / \mathrm{dl}$ o diagnóstico previo de diabetes mellitus tipo 2, colesterol HDL $\leq 40 \mathrm{mg} / \mathrm{dl}$ en hombres o $\leq 50 \mathrm{mg} / \mathrm{dl}$ en mujeres, o que esté en tratamiento para esta anormalidad, triglicéridos $\geq 150 \mathrm{mg} / \mathrm{dl}$ o que se encuentre en tratamiento para esta anormalidad y tensión arterial sistólica $\geq 130 \mathrm{mmHg}$ o diastólica $\geq 85 \mathrm{mmHg}$ o tratamiento previo para hipertensión arterial diagnosticada). Esta condición se encontró en el $25.4 \%$ de los hombres $(n=54)$ y $4.6 \%$ de las mujeres $(n=6)$. Por último se estableció por el índice Framingham un nivel de riesgo intermedio de $27.7 \%$ en la población masculina $(n=59)$ y $4.6 \%$ la femenina $(n=6)$. Un $3.3 \%(n=7)$ del total se estratificó en riesgo alto cardiovascular correspondiente solo a población masculina (Tabla 2).

También se hizo un análisis de correspondencias múltiples cuyos resultados se ven en la Figura 1. Los sedentarios se caracterizan por ser estudiantes, mujeres y especialidades quirúrgicas en mayor proporción.

\section{Discusión}

En este estudio la tasa de participación fue más alta en los estudiantes de posgrado que en los docentes (67.3\% y $32.7 \%$ ). La principal dificultad que explica esta cifra, es que olvidaban o se les dificultaba llegar en ayunas para realizar la toma de los paracínicos, lo cual limitó la tasa de respuesta, pero de todas formas en estos tipos de estudio en la población general, según la literatura, es una adecuada respuesta. ${ }^{8}$

La edad promedio fue de 34 años que se explica por el mayor porcentaje de estudiantes (67\%). Los resultados en cuanto sobrepeso y obesidad medidos a partir del cálculo de índice de masa corporal (sobrepeso $\geq 25 \mathrm{y}<$ $30 \mathrm{k} / \mathrm{m}^{2}$, obesidad $\geq 30 \mathrm{k} / \mathrm{m}^{2}$, muestran una prevalencia de $36 \%$ y $7 \%$, que al compararlos con los referentes nacionales ${ }^{9}$ de 32 y $14 \%$ significa que la población del presente estudio tiene una prevalencia más alta en sobrepeso que en obesidad, en comparación con el nivel nacional. A su vez la prevalencia de la obesidad abdominal en este estudio es de $36 \%$ que expresa la estrecha relación como predictor de enfermedad coronaria. ${ }^{10}$ 


\begin{tabular}{|c|c|c|c|c|}
\hline & \multicolumn{2}{|c|}{ Hombres } & \multicolumn{2}{|c|}{ Mujeres } \\
\hline 。 & $n=213$ & $(61.7 \%)$ & $n=132$ & $(38.3 \%)$ \\
\hline Edad (promedio DE) & 36 & $(9.9)$ & 31 & $(6.2)$ \\
\hline mínimo - máximo & 25 & 76 & 24 & 59 \\
\hline \multicolumn{5}{|l|}{ Factores de riesgo } \\
\hline $\begin{array}{l}\text { Perímetro abdominal, cm } \\
\text { (promedio, DE) }\end{array}$ & 89.3 & $(10.0)$ & 73.4 & $(7.1)$ \\
\hline mínimo - máximo & 59 & 120 & 59 & 102 \\
\hline obesidad abdominal IDF, n (\%) & 97 & $(45.5)$ & 19 & (14.4) \\
\hline \multicolumn{5}{|l|}{ IMC } \\
\hline bajo peso, n(\%) & 0 & $(0.0)$ & 1 & $(0.76)$ \\
\hline normopeso, n(\%) & 87 & $(40.8)$ & 108 & $(81.8)$ \\
\hline sobrepeso, n(\%) & 103 & $(48.4)$ & 21 & (15.9) \\
\hline obesidad, n(\%) & 23 & $(10.8)$ & 2 & $(1.5)$ \\
\hline \multicolumn{5}{|l|}{ Lípidos } \\
\hline $\begin{array}{l}\text { colesterol total } \mathrm{mg} / \mathrm{dl} \\
\text { (mediana, RIQ) }\end{array}$ & 201 & $\begin{array}{l}(183- \\
222)\end{array}$ & 190 & $\begin{array}{l}(167- \\
210)\end{array}$ \\
\hline triglicéridos mg/dl (mediana, RIQ) & 167 & $\begin{array}{l}(111- \\
232)\end{array}$ & 103 & $\begin{array}{l}(74- \\
137)\end{array}$ \\
\hline hipertrigliceridemia, n (\%) & 119 & $(55.8)$ & 27 & (20.6) \\
\hline HDL mg/dl (mediana, RIQ) & 40 & $\begin{array}{l}(36- \\
49)\end{array}$ & 57 & $\begin{array}{l}(47- \\
65)\end{array}$ \\
\hline HDL bajo, n (\%) & 99 & $(46.5)$ & 44 & (33.6) \\
\hline LDL mg/dl (mediana, RIQ) & 120 & $\begin{array}{l}(100- \\
150)\end{array}$ & 110 & $\begin{array}{l}(90- \\
130)\end{array}$ \\
\hline \multicolumn{5}{|l|}{ Presión arterial } \\
\hline sistólica, mm Hg (promedio, DE) & 116 & (12.8) & 107 & $(10.9)$ \\
\hline diastólica, $\mathrm{mm} \mathrm{Hg}$ (promedio, DE) & 79 & $(8.1)$ & 74 & $(7.2)$ \\
\hline \multicolumn{5}{|l|}{ Glicemia } \\
\hline ayunas, mg/dl (mediana, RIQ) & 83 & $\begin{array}{l}(78- \\
89)\end{array}$ & 77 & $\begin{array}{l}(72- \\
82)\end{array}$ \\
\hline alterada de ayuno, $n(\%)$ & 5 & $(2.8)$ & 2 & $(1.5)$ \\
\hline diabetes mellitus $2, n(\%)$ & 1 & $(0.5)$ & 0 & $(0.0)$ \\
\hline Tabaquismo, n (\%) & 39 & $(18.3)$ & 17 & (12.9) \\
\hline Sedentarismo, $n(\%)$ & 162 & $(66.0)$ & 115 & $(87.1)$ \\
\hline Síndrome metabólico, n (\%) & 54 & $(25.4)$ & 6 & $(4.6)$ \\
\hline \multicolumn{5}{|l|}{ Riesgo cardiovascular } \\
\hline latente, $\mathrm{n}(\%)$ & 147 & $(69.0)$ & 125 & (95.4) \\
\hline intermedio, $\mathrm{n}(\%)$ & 59 & (27.7) & 6 & (4.6) \\
\hline alto, n (\%) & 7 & (3.3) & 0 & $(0.0)$ \\
\hline
\end{tabular}

La prevalencia del hipercolesterolemia tomando cifras mayores de $200 \mathrm{mg} / \mathrm{dl}$ fue de $45,5 \%$. El referente nacional con cifras mayores o igual a $240 \mathrm{mg} / \mathrm{dl}$ es de $7,82 \%{ }^{11}$, en contraste con el valor elevado del presente estudio que es de $11 \%$. Para el HDL bajo (<40 mg/dl) la prevalencia nuestra es de $34,4 \%$ y el nivel nacional de $62.8 \%$. Para el HDL alto ( $>$ de $60 \mathrm{mg} / \mathrm{dl}$ ) es de $20,8 \%$ mientras el nacional es de $4,54 \% .^{12}$

En cuanto a la hipertrigliceridemia (triglicéridos $\geq 150$ $\mathrm{mg} / \mathrm{dl}$ ), la prevalencia de este estudio está en $39,4 \%$, la cual no fue medida en la encuesta nacional de salud. De todas formas es alta, puesto que casi cuatro de cada diez personas tienen los triglicéridos altos.

La prevalencia del tabaquismo (se define fumador a todo individuo que consuma tabaco de forma habitual o esporádica, incluyendo el consumo de un cigarrillo en el último mes). En el presente estudio la prevalencia es de $16 \%$, siendo superior al nivel nacional de $12,8 \%$. En esta misma encuesta se discrimina según el nivel de educación, siendo la del universitario y posgrado de $10 \%$, que es inferior al nuestro. ${ }^{13}$ La prevalencia del sedentarismo del estudio fue de $80,2 \%$, la cual se desagregó en estudiantes residentes y docentes ( $84 \%$ y $72 \%$ ), siendo la nacional de $82 \% .{ }^{14}$ Esto quiere decir que según estos resultados la población de más riesgo es la de estudiantes residentes, que excede el promedio nacional.

La prevalencia del síndrome metabólico fue de 17,3\% comparado con otros estudios en diferentes poblaciones que oscila entre $27 \%{ }^{15}, 23,64 \%^{16}$ y $28 \% .{ }^{17} \mathrm{La}$ prevalencia de hipertensión arterial (sistólica $>140$ mm $\mathrm{Hg}$ y diastólica $>90 \mathrm{~mm} \mathrm{Hg}$ ) fue de $13 \%$, mientras el colombiano es de $22.8 \% .{ }^{18}$ La prevalencia del factor de riesgo cardiometabólico según Framingham se puede resumir en la Tabla 3.

En la actualidad es difícil encontrar estudios similares que analicen el riesgo en este tipo de personal (médicos

\begin{tabular}{|l|c|c|c|}
\hline \multicolumn{3}{|c|}{ Tabla 3. Riesgo cardiometabólico según } \\
Framingham (\%)
\end{tabular}




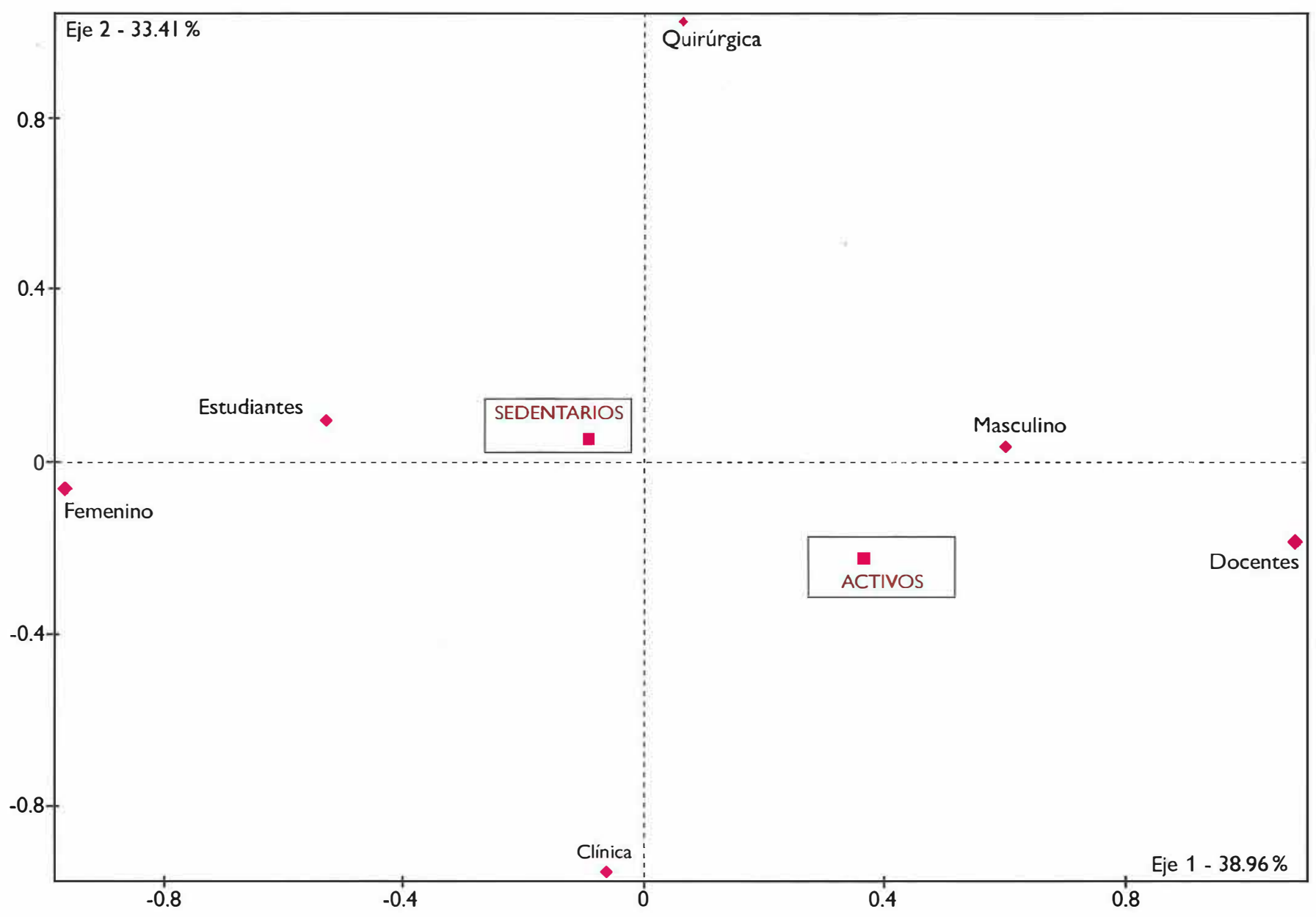

Figura I. Plano factorial, caracterización del sedentarismo según género, vinculación y dependencia.

especialistas y estudiantes de especialización) para poder realizar la comparabilidad. Hay uno hecho en población bogotana de empleados, docentes o familiares que trabajaban en la Universidad Nacional de Colombia, mayores de 18 años, con una muestra de 364 personas. Calcularon el riesgo cardiometabólico con los siguientes resultados: latente $61,8 \%$, intermedio $32,1 \%$ y alto $6,1 \%$. La edad promedio fue de 44 años. ${ }^{19}$ Otro estudio en población general de Medellín cuya muestra fue de 372 personas, revela un riesgo cardiovascular bajo de $81,2 \%$, moderado de $12,1 \%$ y alto de $6,7 \% .^{20}$

Analizando los resultados es preocupante que se presenten cifras más altas que los referentes nacionales en sobrepeso, hipercolesterolemia, tabaquismo, sedentarismo y riesgo cardiometabólico según Framinghan. Esto expresa la alta prevalencia de factores de riesgo modificables en la población de este estudio, lo cual implica acciones inmediatas en la intervención tanto en políticas públicas como de intervenciones puntuales dentro de la Fundación Universitaria Ciencias de la Salud.

Así, el presente estudio es pionero dentro de su clase que no solo calculó el riesgo cardiometabólico en docentes de medicina y en residentes de diferentes especialidades, sino que su principal objetivo es que a partir de los presentes resultados se puedan proponer acciones políticas dentro de la universidad para el mejoramiento y promoción de la salud cardiovascular y de esta manera realizar acciones de prevención primaria, secundaria y terciaria.

Limitaciones: la dificultad para obtener una tasa de respuestas superior al $90 \%$ de los docentes y residen- 
tes limitó detallar los comportamientos entre las dos poblaciones y orientar la toma de decisiones. De todas formas, los resultados servirán como insumo para cambiar y fortalecer políticas institucionales como la de universidad saludable.

Conclusiones: se evidencia alta prevalencia de factores de riesgo cardiometabólico modificables. Es necesario sensibilizar a la población médica de la FUCS y sus directivos sobre la importancia de generar políticas que promuevan espacios para modificar hábitos orientados a la promoción de estilos de vida saludables, el fomento de cambios culturales por medio de la educación en la responsabilidad del autocuidado y dar ejemplo a los pacientes.

\section{Referencias}

1 Rodgers A. The Word Health Report: Reducing Risks, Promoting Healthy Life. Geneva- Switzerland: World Health Organization; 2002.

2. Departamento Administrativo Nacional de Estadistica (DANE). Registro de Defunciones y Nacimientos del año 2005. DANE. Estimaciones 1985-2005 y Proyecciones 2006-2020 anualizadas por sexo y edad. Departamento Admonistrativo Nacional de Estadisticas (DANE); 2006.

3. Rincón OS, Gamarra G, Jerez H, Vera LM. Valoración del riesgo cardiovascular global y prevalencia de síndrome metabólico en trabajadores de la salud del Hospital Universitario Ramón González Valencia. Acta médica.colombiana 29[4], 312-321. 2004

4. Schargrodsky H, Hemandez-Hemandez R, Champagne BM, Silva H, Vinueza R, Silva Aycaguer LC, et al. CARMELA: assessment of cardiovascular risk in seven Latin American cities. Am J Med 2008 Jan;121(1):58-65.

5. Organización Panamericana de la salud. Estadística de Salud de la Américas: Mortalidad. Washington, D.C.: Organizacion Panamericana de la Salud; 2006.

6. Frank E, Tong E, Lobelo F, Carrera J, Duperly J. Physical activity levels and counseling practices of U.S. medical students. Med Sci Sports Exerc 2008 Mar;40(3):413-21.
7. Lobelo F, Duperly J, Frank E. Physical activity habits of doctors and medical students influence their counselling practices. Br J Sports Med 2009 Feb;43(2):89-92.

8. ArancetaJPerez C. Foz M, Mantilla T, Serra Ll, Moreno B, Monereo S, Millan J. Tablas de evaluación del riesgo coronario adaptadas a la población española. Estudio DORICA.Medicina Clínica 2004;123(18):686-91.

9. Rodríguez J, Ruiz F, Peñaloza E, Eslava J, Gómez LC, Sánchez H, Amaya JL, Arenas R, Botiva Y. Encuesta Nacional de Salud 2007. Resultados Nacionales. Ministerio de la Protección Social. Colciencias. Pontificia Universidad Javeriana. Sistemas Especializados de Información. Cendex. ISBN 978-958-716-187-8.

10. Gonzalez A, Urena J, Lavielle M. Chassin O, Elizondo A. Hemandez H. Comparación de índices antropométricos como predictores de riesgo cardiovascular y metabólico en población aparentemente sana. Resvista Mexicana de Cardiologia Vol 22 No 2 Abril-Junio 2011. Pp 59-67.

11. RodriguezOpcitpp 162.

12. Ibidpp 163

13. Rodriguez. Opcitpp 152

14. Ibidpp 154

15. Lombo B, Villalobos C, Tique C, Satisabal C, Franco C. Prevalencia del síndrome metabólico entre los pacientes que asisten al servicio Clínica de Hipertensión de la Fundación Santa Fe de Bogotá. Revista Colombiana de Cardiologia. ISNN: 0120-5633, Vol 12 No 72006 Pp 472-478

16. Villegas A, Botero J, Arango I, Arias S, Toro M. Prevalencia del síndrome metabólico en El Retiro, Colombia. Revista Medica IATREIA, Universidad de Antioquia. ISSN 0121-0793. Vol 16, No 4 (2003).

17. Mendivel C, Sierra I, Perez C. Valoración del riesgo cardiovascular global y prevalencia de dislipemias según los criterios del NCEP-ATP III en una población adulta de Bogotá, Colombia. Clinicallnvestigation Arteriosclerosis. 2004. Vol 6 (3). $\mathrm{Pp}$ 99-107

18. RodriguezOpCit, 161

19. Mendevil C, Sierra I, Perez C. Valoracion del riesgo cardiovascular global y prevalencias de dislipidemias según los crieterios del NCEP-ATP IIlen una población adulta de Bogota Colombia. ClinicalInvestigation Arteriosclerosis 2004; $16(3)$ : 99-107.

20. Jaramillo N, Torres Y, Echavarria E, Llamas A, Montoya L, Pareja D. Estudio sobre factores de riesgo cardiovasculares en una población de influencia de la Clinica las americas. Revista CES Medicina. Vol 18, No 2Julio Diciembre 2004. Pp 9-18. 